Noncirrhotic portal hypertension and didanosine: a re-analysis

Young, J ; Klein, M B ; Ledergerber, B

DOI: https://doi.org/10.1093/cid/ciq079

Posted at the Zurich Open Repository and Archive, University of Zurich

ZORA URL: https://doi.org/10.5167/uzh-54424

Journal Article

Published Version

Originally published at:

Young, J; Klein, M B; Ledergerber, B (2011). Noncirrhotic portal hypertension and didanosine: a reanalysis. Clinical Infectious Diseases, 52(1):154-155.

DOI: https://doi.org/10.1093/cid/ciq079 


\section{Noncirrhotic Portal Hypertension and Didanosine: A Re-Analysis}

To the Editor-In 2020, Kovari et al [1] reported a strong association between exposure to didanosine (DDI) and noncirrhotic portal hypertension $(\mathrm{NCPH})$, a rare condition likely to be of multifactorial etiology. However, the authors were not able to control confounding through multivariate modeling because of the small number of case patients. This limitation is typical of a rare condition, making it more difficult to evaluate the association of antiretroviral use with such events because of the number of factors that influence the prescription of these drugs. With only 15 cases, adding even a single confounder to a model may introduce more bias than it removes because of small sample bias away from the null value (an odds ratio of 1) [2]. Their study was, however, nested within the Swiss HIV Cohort Study; thus, other methods of confounder control are available. These methods require additional modeling of DDI use in the wider cohort.

Using logistic regression, we modeled the probability of first use of DDI over time for each patient in the cohort, starting from the month when either infection was first known or DDI was first marketed in Switzerland until the month of either first use of DDI or the end of follow-up. Our model for first use of DDI had a time-dependent intercept based on a cubic spline and covariates of sex, ethnicity, education, likely transmission group, age, the number of failed regimens (time dependent), and timedependent indicators for hepatitis (chronic B or C), lipoatrophy, diabetes, nervous system toxicity, Centers for Disease Control and Prevention groups $B$ and $C$, use of zalcitabine, use of stavudine, use of tenofovir, gastrointestinal toxicity, and pregnancy and further interaction terms between these last 4 indicators and the time of related warnings issued either by the US Food and Drug 
Administration or the drug company. From this model, we then calculated a propensity score for each patient at each point in time: the probability of exposure to DDI, given the patient's covariate and treatment history up to that point. This probability is related to an inverse probability of treatment weight (IPTW) (see Appendix 1 in [3]) and can therefore be calculated in a similar way (see Appendix in [4]).

We then re-analysed the original 15 case patients and 75 matched control subjects, adjusting for a single covariate: the propensity score at the date of diagnosis in the case patient. This means that case and control exposures were compared at a common value of the propensity to be exposed to DDI. We made this comparison at a common value of the log-transformed propensity score; with a log transformation, both exposed and unexposed patients had propensity scores with a similar variance, as is necessary for this method of adjustment [5]. Finally, we added prior information to our re-analysis. With only a few matched sets, small sample bias can be severe even when exposure is the only variable in the model [6]. Adding prior information can limit this bias by assigning essentially zero prior probability to clinically implausible values of an estimate. One of us (MBK), a clinician with expertise in liver disease and HIV infection, having read other case reports and series (see Table 1 in [1]) and before reading about this study, asserted her opinion that the odds of $\mathrm{NCPH}$ in exposed patients, compared to those unexposed, was a ratio of 1.2 per year of exposure to DDI, with a $95 \%$ confidence interval (CI) of .5-2.5. We generated a set of matched case-control pairs to represent this prior opinion and then reran the analysis using both prior and real data $[7,8]$.

The published unadjusted odds ratio for a year of exposure to DDI is 3.4 (95\% CI, 1.5-8.1) [1]. After adjustment using the $\log$ propensity score, our estimate was 4.0 (95\% CI, 1.2-13); a weighted analysis using IPTWs gave an estimate of 4.7 (95\% CI, 1.4-16). In the Bayesian analysis of prior and real data, the adjusted estimate was 2.2 (95\% CI, 1.5-3.3).

Propensity scores and IPTWs are ideal methods of confounder control if the outcome is rare but treatment is common [9]. The 2 methods use very different statistical logic; that both lead to a similar estimate is reassuring. Propensity scores have a Bayesian interpretation [5]; thus, we used this method for our Bayesian analysis. Our re-analysis showed how even a large number of factors that potentially influence treatment allocation can be accounted for in the analysis of a rare outcome. The strong association between DDI and NCPH reported by Kovari et al [1] does not appear to be an artifact of inadequate confounder control. However, the published estimate is probably an over-estimate to some degree, because of small sample bias. That said, there is sufficient evidence in these data to convince a knowledgeable clinician that the association may be of an order of magnitude ( of $\geqslant 2$ ) to justify the Food and Drug Administration warning in January 2010 of an increased risk of $\mathrm{NCPH}$ among patients exposed to DDI [10].

\section{Acknowledgments}

We thank the Swiss HIV Cohort Study and Helen Kovari for the use of cohort and casecontrol data.

\section{Funding. None reported.}

Potential conflicts of interest. M.B.K. has been a consultant for GlaxoSmithKline/Viiv; has received grants from Merck, Canadian Institutes of Health Research, National Institutes of Health Research, and Fonds de recherches en sante du Quebec; has served on the speakers' bureau for Bristol-Myers Squibb, and GlaxoSmithKline/Viiv; has developed presentations for Gilead, GlaxoSmithKliine/ Viiv; and has had meeting expenses paid by GlaxoSmithKline/Viiv. B.L. has served on the board for Tibotec and the speakers' bureau for Tibotec, Roche, and Gilead (paid to institution); and has had meeting expenses paid by Bristol-Myers Squibb (paid to institution). J.Y.: no conflicts.

\section{Jim Young, ${ }^{1}$ and Marina B. Klein, ${ }^{3}$ \\ Bruno Ledergerber ${ }^{2}$}

${ }^{1}$ Basel Institute for Clinical Epidemiology and Biostatistics, University Hospital Basel, Basel,

${ }^{2}$ Division of Infectious Diseases and Hospital

Epidemiology, University of Zurich, Zurich, Switzerland, and ${ }^{3}$ Division of Infectious Diseases and Immunodeficiency Service, McGill University Health Centre, Montreal, Canada

\section{References}

1. Kovari H, Ledergerber B, Peter U, et al. Association of noncirrhotic portal hypertension in HIV-infected persons and antiretroviral therapy with didanosine: a nested case-control study. Clin Infect Dis 2009; 49:626-635.

2. Greenland S, Schwartzbaum JA, Finkle WD. Problems due to small samples sparse data in conditional logistic regression analysis. Am J Epidemiol 2000; 151:531-539.

3. Robins JM, Hernan MA, Brumback B. Marginal structural models causal inference in epidemiology. Epidemiology 2000; 11: 550-560.

4. Hernan MA, Brumback B, Robins JM. Marginal structural models to estimate the causal effect of zidovudine on the survival of HIVpositive men. Epidemiology 2000; 11: 561-570.

5. Rosenbaum PR, Rubin DB. The central role of the propensity score in observational studies for causal effects. Biometrika 1983; 70:41-55.

6. Greenland S. Small-sample bias corrections for conditional maximum-likelihood oddsratio estimators. Biostatistics 2000; 1 : $113-122$.

7. Greenland S, Christensen R. Data augmentation priors for Bayesian semi-Bayes analyses of conditional-logistic and proportional-hazards regression. Stat Med 2001; 20:2421-2428.

8. Greenland S. Prior data for non-normal priors. Stat Med 2007; 26:3578-3590.

9. Braitman LE, Rosenbaum PR. Rare outcomes, common treatments: analytic strategies using propensity scores. Ann Intern Med 2002; 137:693-695.

10. US Food and Drug Administration. FDA drug safety communication: serious liver disorder associated with the use of Videx/Videx EC (didanosine) Available at: http://www.fda.gov/ Drugs/DrugSafety/PostmarketDrugSafety InformationforPatientsandProviders/ucm 199169.htm. Accessed 31 August 2010.

Correspondence: Dr Jim Young, Basel Institute for Clinical Epidemiology and Biostatistics, Hebelstrasse 10, University Hospital Basel, CH-4031 Basel, Switzerland (jyoung@ uhbs.ch).

Clinical Infectious Diseases 2011;52(1):152-155

(C) The Author 2011. Published by Oxford University Press. All rights reserved.

1058-4838/2011/521-0001\$37.00

DOl: $10.1093 /$ cid/ciq079 